\title{
KASS v.2.2. Scheduling Software for Construction with Optimization Criteria Description ${ }^{\dagger}$
}

\author{
M. KRZEMIŃSKI* \\ Warsaw University of Technology, Civil Engineering Department, Warsaw, Poland
}

\begin{abstract}
The paper presents fourth version of specialist useful software in scheduling KASS v.2.2 (Algorithm Scheduling Krzeminski System). KASS software is designed for construction scheduling, specially form flow shop models. The program is being dedicated closely for the purposes of the construction. In distinguishing to other used programs in tasks of this type operational research criteria were designed closely with the thought about construction works and about the specificity of the building production. The minimal time, the minimal slack of brigades, the minimal slacks of the chosen working brigade and costs of the transfer operation of working fronts are included in operational research criteria between work centers. It is possible to enter data into the program both by hand as well as to load the Excel from files, similarly is with results, they are presented on-screen as well as a possibility of enrolling them in the existing file Excel. An element is very valid for it since allows for further simple processing of received results. In providing software for performing operational research calculations a technique of the complete review and simulation technology are being exploited. A program describing algorithms is used which will stay in the article as well as shown computational examples will remain.
\end{abstract}

DOI: 10.12693/APhysPolA.130.1439

PACS/topics: $89.20 . \mathrm{Kk}, 89.75 . \mathrm{Fb}$

\section{Introduction}

Construction scheduling problem is particularly important when the work accomplishment process is being prepared. Scheduling is a fast-growing area of scientific research in construction production engineering. It has applied methods of artificial intelligence, such as genetic algorithms, and colony optimization algorithms, simulated annealing, neural networks. But also fuzzy logic techniques/elements of fuzzy logic are being widely used in order to determine time $[1,2]$.

This paper investigated a flow-shop scheduling problem, which consists in determining the optimum working sequence for brigades at workspaces. The model is based on assumptions that it is necessary to perform a sequence of technological operations, that the sequence is determined in advance and cannot be changed, that every brigade works at chosen workspace only once and at the exact time of its transition to another workspace, the next brigade comes in $[3,4]$.

There is a lot of computer software which can help with scheduling process. Most popular are MS Project and Primavera. If we looking for software dedicated for flow shop models we can find programs like AIMMS Flow Shop [5] and LEKIN - Scheduling System [6]. These are very good and efficient programs but for mass production. For unique construction production we need something different. The construction has special conditions during production process so software needs to have

*e-mail: m.krzeminski@il.pw.edu.pl

${ }^{\dagger}$ Paper based on contribution to 2nd International Conference on Computational and Experimental Science and Engineering (ICCESEN 2015) number of specified assessment criteria to give the best result. Answer for this problem may be KASS software.

\section{Criteria for scheduling}

There is a lot of criteria for scheduling for construction scheduling. Developed in this section is a set of criteria that are the base for a model of the complete review scheduling $[1,7]$. Below you will find the description of the developed criteria.

The shortest lead time is understood as the smallest length of a schedule. The criterion often appears in the optimization tasks from the scope of the scheduling. It is particularly important for the construction industry, because it allows one to specify the deadline of all the work.

Introduction of this criterion in the dual form seems to be reasonable. First a classic form, concerning the implementation of all steps in all working centres. Second dedicated for the construction industry, concerning the implementation of a certain number of steps (counting from the beginning) of the entire production cycle in all working centres. The division is proposed due to the volatility in the construction industry, which often connects with changes of subcontractors for the work.

In the case of searching for the shortest term for the entire schedule, one must use the following relationship:

$$
T_{\min }=\min _{x \in(1, u)}\left(T_{n m}^{k, x}\right),
$$

where $u$ - number of consecutive schedule variants, $n-$ number of working brigades, $m$ - number of working centres, $\mathrm{T}_{\mathrm{nm}}^{\mathrm{k}} T_{n m}^{k}$ - due date of the last step in selected schedule $p$.

In the case of searching for the shortest due date of all work of the $i$-th selected brigade, the dependence below should be applied 


$$
T_{\text {min }}^{i}=\min _{x \in(1, u)}\left(T_{i m}^{k, x}\right),
$$

where $T_{\mathrm{nm}}^{\mathrm{k}} T_{i m}^{k}$ - due date of the last step of $i$-th brigade in selected schedule $p$.

In the case of the application of this criterion, the schedule with the smallest value of the total work time will be selected as the best. This assumption applies to both the overall and to the selected steps option.

\subsection{The continuity of the work brigades}

The continuity of the work brigades is one of the most important criteria used in construction in the scheduling process. Each owner of the company is dependent on the possession of well qualified and harmonious team. One of the elements that allow you to achieve this goal is to ensure an adequate level of compensation for their employees. The compensation flows partly from the work done by the brigade. We can conclude that to have a good team, one must ensure the greatest possible continuity in the work. The continuity of the work also allows for easier development of harmony in the team.

Also in this case, it seems reasonable to make a certain detail. Basically, it can be assumed that decisive will be a minimal downtime of all brigades. Also proposed is the possibility of applying this criterion to one or more selected brigades. Such an assumption is especially relevant in the case of having subcontractors to selected steps.

The continuity of the work brigades can be determined by selecting the solution with the smallest value of the downtime of a working brigade. If one wants to perform an optimization with respect to selected $i$-th brigade, a relationship can be applied

$$
C P B_{i, \min }=\min _{x \in(1, u)}\left(\left(\sum_{j=1}^{m} T_{i, j}^{p}-T_{i, j-1}^{k}\right)^{(x)}\right)
$$

where $T^{p}$ - date of the start of work of the working brigade in working centre, $T^{k}$ - date of the end of work of the working brigade in working centre, $i$ - number of working brigade, $j$ - index of working centre.

It is also possible to optimize execution relative to the minimum of the total downtime of all brigades $C P B_{\min }$. To do this, one can use the following relationship:

$$
C P B_{\min }=\min _{x \in(1, u)}\left(\sum_{y=1}^{i}\left(\sum_{j=1}^{m} T_{y, j}^{p}-T_{y, j-1}^{k}\right)^{(x)}\right) .
$$

When calculating a schedule with minimal downtime of all brigades, also applied can be a weight system. Every brigade must be assigned a weight $\mathrm{w}_{\mathrm{i}}$. The calculation should be based on the following:

$$
\begin{aligned}
& C P B W_{\min }= \\
& \min _{x \in(1, u)}\left(\sum_{y=1}^{i} w_{y}\left(\sum_{j=1}^{m} T_{y, j}^{p}-T_{y, j-1}^{k}\right)^{(x)}\right),
\end{aligned}
$$

where $w$ - impact of work of selected working brigade and $\sum_{x=1}^{n} w_{x}=1$.
You can also designate an optimal schedule relative to, for example, two brigades, both with and without weights. To do this, you must modify the above dependences by limiting the scope of their activities to selected working centres.

In the case of the application of this criterion, a schedule with the smallest value of total downtime of brigades will be selected as the best. This assumption applies to both the overall and to the selected steps option.

\subsection{Term unexceedableness}

Term unexceedableness is another criterion, but not less important than the previous ones. One of the essential elements of the agreement on the execution of construction works is the due date of the work. This is the deadline, and when overrun, may entail the need to incur any contractual penalties.

The criterion may be used as the directive term of the whole investment, as well as the unexceedable term of the completion of works. In this case, at the stage of data input to the optimization tasks, one must provide not only the directive term of the whole process, but also the dates for individual working centres. You can assign all or only selected centres.

The criterion can also be applied so that the deadline applies only to the work of chosen working brigade. We are here unable to shorten the time that is required, and results from amount of work and selected technologies.

In the case of computation for the entire schedule, determine the deadline for directive term of investment $T D$ and then check next solutions in terms of meeting the following condition:

$$
T D \geq T_{n m}^{k} .
$$

By applying the criterion of the deadline for completion of the works by the working brigade, enter the term directive $T D_{i}$ completion by the working brigade and make use of the following:

$$
T D_{i} \geq T_{i m}^{k} .
$$

There is also the possibility to introduce the directive term for the completion of work on the selected plot of $\mathrm{TD}_{j}$. Working condition for such a task set is as follows:

$$
T D_{j} \geq T_{n j}^{k} \text {. }
$$

Another relationship, you can enter the directive term of the completion by the working brigade in the selected working centre $\mathrm{TD}_{i j}$. Relationship, which should be used is listed below

$$
T D_{i j} \geq T_{i j}^{k} \text {. }
$$

In the case where it is not possible to find a solution that meets the individual terms and conditions, one must select a solution the next $\mathrm{TD}_{\mathrm{opt}}$. Such solution can be determined by the following:

$$
T D_{\mathrm{opt}}=\min _{x \in(1, u)}\left(T_{i j}^{k, x}-T D_{i j}\right) .
$$

The above dependences can be entered individually or in the selected configuration. One can enter, for example, a relationship of the directive term of the investment and 
a relationship of the directive term for the completion of work by the Working Party. With a few limitations at the same time TDM is to be reckoned with an option, in which no schedule will be able to meet the conditions. In this case, choose the optimal solution TDMopt deviating in the slightest from complete solution, using the following relationship:

$$
\mathrm{TDM}_{\text {opt }}=\min _{x \in(1, u) y \in(1, a)}\left(\left(T_{i j}^{k, y}\right)^{(x) 1}\right),
$$

where $a-$ number of consecutive restrictions.

It is also possible to make a few restrictions while indicating their validity by entering weights $w_{a}$. It will not matter in the case in which it will be possible to meet all of them. On the other hand, if one encounters a case where not all constraints can be satisfied one must perform calculations based on the following:

$$
\begin{aligned}
& \mathrm{WTDM}_{\mathrm{opt}}= \\
& \min _{x \in(1, u)}\left(\left(\sum_{y=1}^{a} T_{i j}^{k, y}-T D_{i j} w_{y}\right)^{(x)}\right),
\end{aligned}
$$

where $w_{y}$ - impact of work of selected working brigade and $\sum_{x=1}^{n} w_{x}=1$.

In the case of the application of this criterion schedules with unexceeded deadline of directive term will be selected as the best. If, however, there are several possible solutions, one must choose which will allow the biggest worktime "profit". Also, chosen can be the one that provides the greatest possible continuity of work of working brigades.

\subsection{The cost of moving the front of the works}

The cost of moving the front of the works - this is the criterion that has been selected in this work. The cost in this case should be understood not only as a financial effort, but as the effort of all kinds of measures.

The task of the criterion is to influence the task schedule in such a way that the total cost of moving fronts of works would be the smallest. To do that, at the stage of entering data for a task create a series of the cost matrices for the transfer of individual brigades $\left(M K_{i}\right)$ between the fronts. Matrices need not be symmetrical. If, however, the move of a brigade is not a problem, you can make no transition cost matrix. Matrix of costs should be defined as follows:

$$
M K_{i}=\left[\begin{array}{cccc}
0 & k_{12} & \ldots & k_{1 j} \\
k_{21} & 0 & \ldots & k_{2 j} \\
\ldots & \ldots & 0 & \ldots \\
k_{j 1} & k_{j 2} & \ldots & 0
\end{array}\right],
$$

where $i$ - index of working brigade, $j$ - number of working centres.

It should be noted that the matrix need not be symmetrical, for example the transition of the brigade from the working centre A to B does not have to cost as much as the transition of the same brigade from B to A. That dependence is reflected inter alia in a large expansion and complexity of construction transport.
Given schedule, one can calculate the cost of transition of a brigade KBi between working centres. Select the solution with the lowest cost of brigade transition per the following relationship:

$$
K B_{i}=\min _{x \in(1, u)}\left(K B_{i}^{(x)}\right) .
$$

In the case in which we will give more than one cost matrix the costs of the transition of the individual brigade must be summed up with the following equation and choose the solution giving the smallest value:

$$
K B=\min _{x \in(1, u)}\left(\left(\sum_{y=1}^{n} K B_{y}\right)^{(x)}\right),
$$

where $n$ - number of consecutive working centres.

It is also possible to enter the weights for the transition costs of individual brigades. Such a case is presented by the following relationship:

$$
W K B=\min _{x \in(1, u)}\left(\left(\sum_{y=1}^{n} K B_{y} w_{y}\right)^{(x)}\right),
$$

where $w_{y}$ - impact of transition of selected working brigades, $\sum_{x=1}^{n} w_{x}=1$.

The criterion should be used as a supplementary criterion [8] or the criterion of less weight. The specificity of the construction industry is that the previously mentioned criteria seem to be much more important.

In the case of the application of this criterion a schedule with the smallest transition cost of the brigades will be selected as the best. This assumption applies to both the overall and to the selected steps option.

\section{KASS V.2.2 program description}

The KASS v.2.2 software was written in the Java programming environment. It has a bilingual (Polish and English) interface, it does not require installation, it can be downloaded for free from the website www.ipb.edu.pl (bookmark - Programy).

The program uses the complete overhaul, when no more than 14 workspaces are considered. If there are more workspaces, the simulation was applied.

There are four optimization criterion: time, continuity, continuity of chosen brigade and brigade transition costs.

To show how the program works, we will use the example of optimization for chosen task. The picture below depicts the welcome window that appears after we start the program.

After we press the button Next, we move to the next window and we must choose workspace count (seven in this example), as well as working brigades count (four in this example).

It is also necessary to choose the assessment criteria which will be taken into account during optimization calculations. It is possible to choose from one to four criteria. In this example shortest work accomplishment time was chosen as the main criterion, continuity is second criterion, continuity of the brigade number 2 is the 


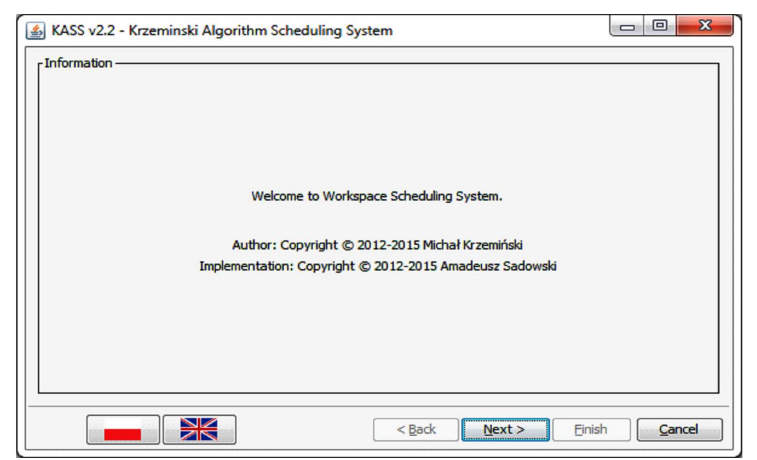

Fig. 1. The welcome window of the KASS v.2.2 program.

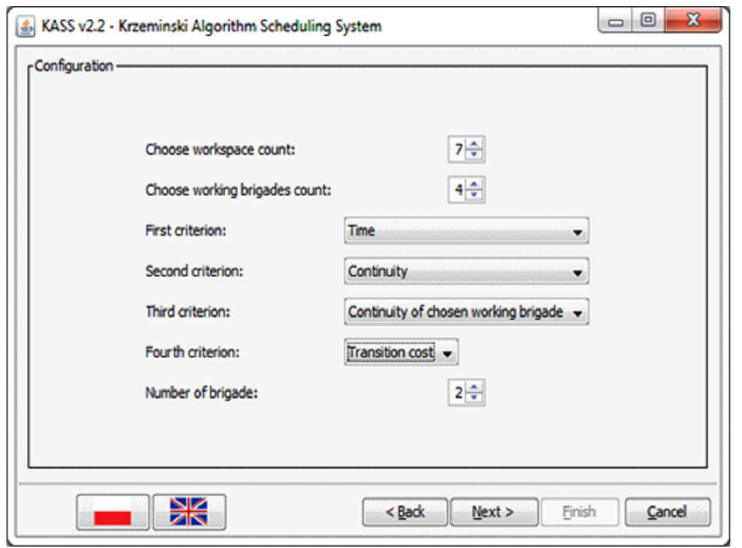

Fig. 2. The configuration window of the KASS v.2.0 program.

third criterion and the lowest transition costs is the last criterion. The criteria sequence is important, as it sets its precedence. The first one will influence the most the results of optimization.

The last picture depicts the results. The window presents the right scheduling and it is also possible to read off data concerning total time, total brigades work stoppages, chosen brigade work stoppage and brigades transition cost.

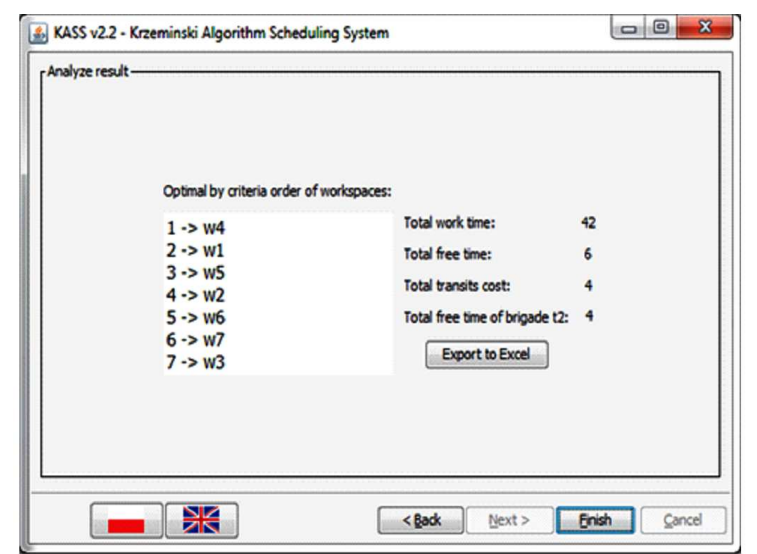

Fig. 3. The results window of the KASS v.2.2 program.
It is also possible to save the results as *.xlsx files, which are readable in the Excel program among others. It is useful, because it enables the KASS v.2.2. users to work further in an easy and comfortable manner on data that were obtained.

\section{Conclusion}

The article presents the latest version of the specialist software KASS. Software is dedicated for construction scheduling. Used assessment criteria and the possibility of cooperation with the MS Excel environment make program appropriate to support the real construction scheduling process. Previous versions of the software were wildly tested. Tests were conducted for finishing works in the mall [7], for roadworks [9] and for monolithic construction works [8].

The author plans to work further on the KASS software and enlarge criteria, introduce heuristics, as well as elaborate the probabilistic version.

\section{References}

[1] N. Ibadov, J. Kulejewski, M. Krzemiński, AIP Conf. Proc. 1558, 1298 (2002).

[2] M. Krzemiński, AIP Conf. Proc. 1648, 600005 (2015).

[3] M.L. Pinedo, Scheduling: Theory, Algorithms, and Systems, Springer, New York 2012.

[4] M.L. Pinedo, Planning and Scheduling in Manufacturing and Services, Springer, Dordrecht 2009.

[5] E. Zondervan, T.P.J. van Boekel, J. Fransoo, A.B. de Haan, Comput. Aided Chem. Eng. 29, 925 (2011).

[6] A. Madureira, I. Pereira, N Sousa, P. Avila, J. Bastos, in: Computational Intelligence for Engineering Systems, Vol. 46 of the series Intelligent Systems, Control and Automation: Science and Engineering, 2010, p. 34 .

[7] M. Krzemiński, E. Krasnodębska, in: Theoretical Foundations Of Civil Engineering Polish-Ukrainian Transactions, Vol. 22, Warsaw 2014, p. 189 (in Polish).

[8] P. Kowalik, M. Krzemiński, Logistyka, 4218 (2015) (in Polish).

[9] M. Krzemiński, A. Wypysiak, Proced. Eng. 91, 400 (2014). 